\title{
EXPERIMENTAL IDENTIFICATION OF AN ELASTO-MECHANICAL MULTI-DEGREE-OF-FREEDOM-SYSTEM USING STOCHASTIC SIGNALS
}

\author{
Simon Schleiter*, Okyay Altay, Sven Klinkel \\ RWTH Aachen University, Faculty of Civil Engineering, Chair of Structural Analysis and Dynamics, \\ Mies-van-der-Rohe-Str. 1, 52074 Aachen,Germany \\ * corresponding author: schleiter@lbb.rwth-aachen.de
}

\begin{abstract}
The determination of dynamic parameters are the central points of the system identification of civil engineering structures under dynamic loading. This paper first gives a brief summary of the recent developments of the system identification methods in civil engineering and describes mathematical models, which enable the identification of the necessary parameters using only stochastic input signals. Relevant methods for this identification use Frequency Domain Decomposition (FDD), Autoregressive Moving Average Models (ARMA) and the Autoregressive Models with eXogenous input (ARX). In a first step an elasto-mechanical mdof-system is numerically modeled using FEM and afterwards tested numerically by above mentioned identification methods using stochastic signals. During the second campaign, dynamic measurements are conducted experimentally on a real 7-story RC-building with ambient signal input using sensors. The results are successfully for the relevant system identification methods.
\end{abstract}

KEYWORDS: System identification, civil engineering structures, stochastic signal, ambient signal, FDD, ARMA, ARX.

\section{INTRODUCTION}

Starting with the $19^{\text {th }}$ century, aesthetic and economic challenges have induced an impulsive interest on the design of the dynamic behavior of civil engineering structures, as the new constructions are getting higher and slenderer. Hence the calculation of relevant system parameters, such as natural frequencies, damping ratios and modal shapes, become more important. In many cases it is necessary to identify system parameters after construction, so that the use of experimental methods becomes essential to get the current behavior. Generally, all experimental methods can be distinguished between either time or frequency domain. The investigated models in this paper are the Frequency Domain Decomposition (FDD) in frequency domain, Autoregressive Moving Average Models (ARMA) and the Autoregressive Models with eXogenous input (ARX), both in time domain.

To describe the system response accurately, the output data should be known as well as the excitation data. But in most cases the determination of the excitation data is very costly for civil engineering structures. For instance, the detection of the wind induced excitation or microseism requires a simultaneous measurement using several sensors. Whereas the ARX-model is using excitation data in the calculation, the ARMA-model, as well as the FDD are neglecting any excitation signal. In this case an assumption must be made. The excitation may only be a broad band excitation, otherwise there is no interpretation of the results possible. Especially for this case the
FDD has been developed by Brincker [1]. For complex structures the use of ARMA-model and FDD are recommendable and mostly unavoidable.

A numerical implementation of all presented models is possible and is executed in this paper. Results are validated on the basis of two examples: one on a numerically simulated 4-DOF system and the other one on an experimentally analyzed 7 -story real structure. Aim of these applications is to show upcoming problems for the presented system identification methods and potential for further research on this topic.

\section{System Identification Methods: FDD, ARMA, ARX}

The Frequency Domain Method (FDD) is based on the spectral power density, which can be used to describe signals in frequency domain. It is approved that the spectral density of the excitation signal $\left(G_{x x}\right)$ and of the response signal $\left(G_{y y}\right)$ can be decomposed by the Frequency Response Function $(H)$, all terms depend on the circular natural frequency and the exponent ${ }^{*}$ illustrates the complex conjugate tensor as ${ }^{T}$ represents the transpose, as follows:

$$
G_{y y}(\omega)=H^{*}(\omega) G_{x x}(\omega) H^{T}(\omega)
$$

Using some mathematical transformations and the assumption of broad band excited systems a single value decomposition of this formulation yields the modal shapes and the natural frequencies of the system. For further information the lecture of Brincker [1] is recommended. Damping ratios can be calculated 


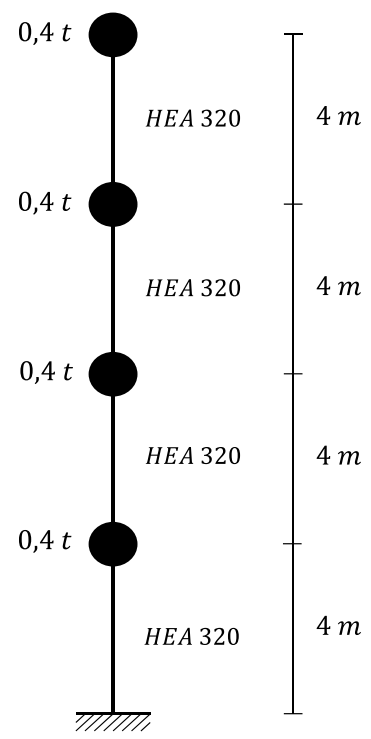

Figure 1. Structure of the 4-dof system.

by extensions of this system, such as Enhanced FDD (EFDD) or Curve Fitting FDD (CFDD) explained in Brincker et al. 22 and Jacobsen et al. 3].

Based on the time domain, the aim of ARMA- and ARX-models is to recreate the time signal by correlation coefficients. This correlation coefficients or parameters can describe the complete system behavior with the assumption of periodic behavior. Additionally, it is possible to create the Frequency Response Function (FRF) for each signal by knowing these parameters. Using this FRF, working from now on in the frequency domain, the modal shapes can be calculated by an algorithm as well as the natural frequencies. Damping ratios are calculated by the so called half power method, mentioned in $\mathrm{He}$ et al. 4, also using the above-mentioned FRF. A detailed overview of these and more models based on time domain is given in Ljung [5] and a closer explanation of the functionality of the FRF in He et al. 44.

\section{Application Examples}

To validate these methods, a numerical application of a 4-dof system (see figure 1) was conducted. For this a cantilever beam was simulated in a FE-program with four similar masses distributed in the same distance to each other. To simulate a measurement, ambient input signals were generated and afterwards used as excitation signals for the structure. By using the numerical response signals, the methods were applied. As a validation of the methods, an identification in the FE-program itself using modal analysis gave the natural frequencies and the damping ratio shown in Table 1 and 2 illustrated as FE-values. The results of the FE-calculation (modal analysis) are in good agreement to the used identification methods (ARMA, ARX, FDD).

The modal shapes are shown in figure 2 , where the
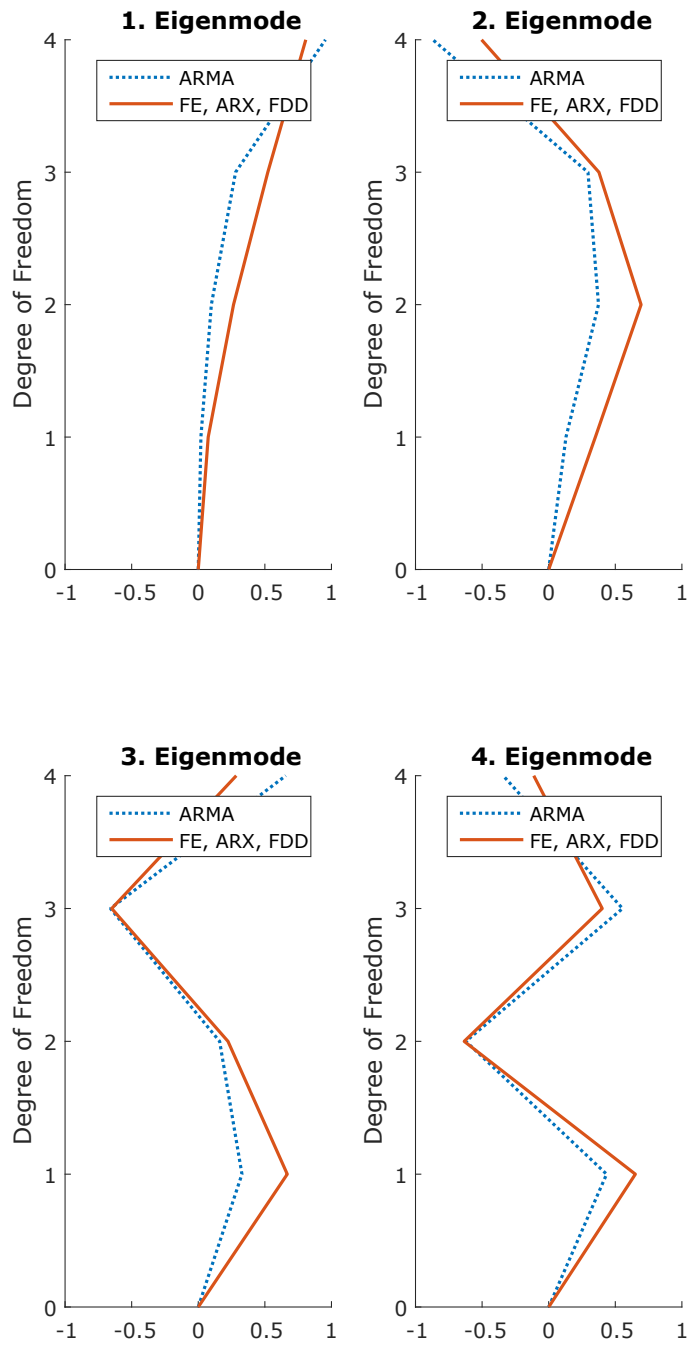

Figure 2. Comparison of the modal shapes of the 4-dof system.

reference modal shapes can be compared with the modal shapes of the different applications. Except the ARMA-model the applications give exactly the same results compared to the FE-reference values. In contrast a certain percentile difference for the ARMAmodel can be realized. It only can describe the modal shapes in a rough shape and could only be accepted for preliminary design purposes.

In a second application a first plausibility check of FDD has been conducted experimentally, based on real measurement data. In this example a 7-story RC-building (see figure 3) is investigated. The measurement data is acquired by using three identical bi-directional geophones, measuring the velocity (sensitivity of $100 \mathrm{~V} / \mathrm{m} / \mathrm{s}$ ). The measurement goal was the estimation of natural frequencies of the bending mode shapes. Therefore the sensors were located in the sixth 


\begin{tabular}{lllll}
\hline Natural frequency $[\mathbf{H z}]$ & FE & FDD & ARX & ARMA \\
\hline 1. Eigenmode & 1,2 & 1,3 & 1,2 & 1,2 \\
\hline 2. Eigenmode & 7,7 & 8,0 & 7,7 & 7,7 \\
\hline 3. Eigenmode & 19,3 & 20,1 & 19,3 & 19,3 \\
\hline 4. Eigenmode & 28,4 & 29,7 & 28,4 & 28,5 \\
\hline
\end{tabular}

TABLE 1. Comparison of the natural frequencies of the 4-dof system.

\begin{tabular}{lllll}
\hline Damping ratio [\%] & FE & FDD & ARX & ARMA \\
\hline 1. Eigenmode & 1,0 & - & 1,0 & 0,9 \\
\hline 2. Eigenmode & 1,1 & 1,5 & 1,1 & 1,1 \\
\hline 3. Eigenmode & 1,9 & 1,9 & 2,2 & 2,1 \\
\hline 4. Eigenmode & 2,5 & 2,5 & 2,7 & 2,7
\end{tabular}

Note: The used algorithm according to the FDD makes a determination of the damping ratio for low frequencies impossible and thus for the first eigenmode.

TABLE 2. Comparison of the damping ratios of the 4-dof system.

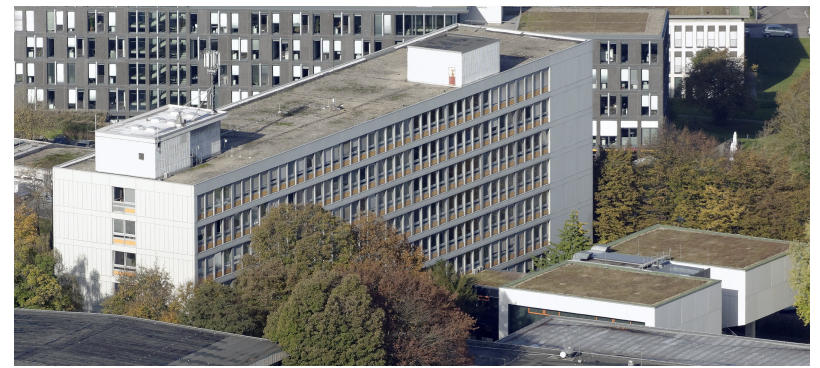

FiguRE 3. Experimentally Investigated 7-story RCbuilding. 6]

story on one axis and a distribution over the short building side was not necessary (see figure 4). A detailed system identification only would be possible by using more measurement points. In this application the FDD method only investigates the applicability for complex civil structures and possible upcoming problems. Load-bearing walls of the structure are illustrated in red in figure 4

An interaction of the $\mathrm{x}$-direction with the $\mathrm{y}$ direction is expectable for some modes, but for the sake of simplicity the modes are investigated separate for each direction. Table 3 shows the results of natural frequencies and damping ratios for the $\mathrm{x}$-direction and Table 4 according to the $y$-direction. The results show that there is an interaction caused by non-symmetry and varied stiffness contribution between the second modes of $\mathrm{x}$ - and $\mathrm{y}$-direction.

The significant variation of the damping ratio down to $0.5 \%$ for the second eigenmode can be interpreted as that it is probably not a bending mode shape like the first and the third eigenmodes. The identified damping ratios are obviously lower than the standard values for RC-buildings indicating the drawbacks of the presented methods for the estimation of the struc-

\begin{tabular}{lll}
\hline & $\begin{array}{l}\text { Natural } \\
\text { Frequency } \\
{[\mathbf{H z}]}\end{array}$ & $\begin{array}{l}\text { Damping } \\
\text { ratio [\%] }\end{array}$ \\
\hline 1. Eigenmode & 1,9 & 1,2 \\
\hline 2. Eigenmode & 2,8 & 1,3 \\
\hline
\end{tabular}

TABLE 3. Natural frequencies and damping ratios of the $\mathrm{x}$-direction of the RC-building.

\begin{tabular}{lll}
\hline & $\begin{array}{l}\text { Natural } \\
\text { Frequency } \\
{[\mathbf{H z}]}\end{array}$ & $\begin{array}{l}\text { Damping } \\
\text { ratio [\%] }\end{array}$ \\
\hline 1. Eigenmode & 2,1 & 1,2 \\
\hline 2. Eigenmode & 2,6 & 0,5 \\
\hline 3. Eigenmode & 4,3 & 2,0 \\
\hline
\end{tabular}

TABLE 4. Natural frequencies and damping ratios of the $\mathrm{y}$-direction of the RC-building.

tural damping. Since these eigenmodes are decoupled the damping ratio can be completely different compared to the bending eigenmodes.

At this stage using the measurement results of the sixth floor, only a preliminary general identification of the eigenmodes in y-direction can be concluded, as in x-direction all measurement points lie on the same axis. A more sophisticated system identification can be done by including additional measurement points, also in vertical direction. The results of the modal shapes by application of the FDD are shown in figure 57 7 It can be seen, that the first and the third modes both can be interpreted as the bending modes and thus the second mode shows a torsional behavior. 


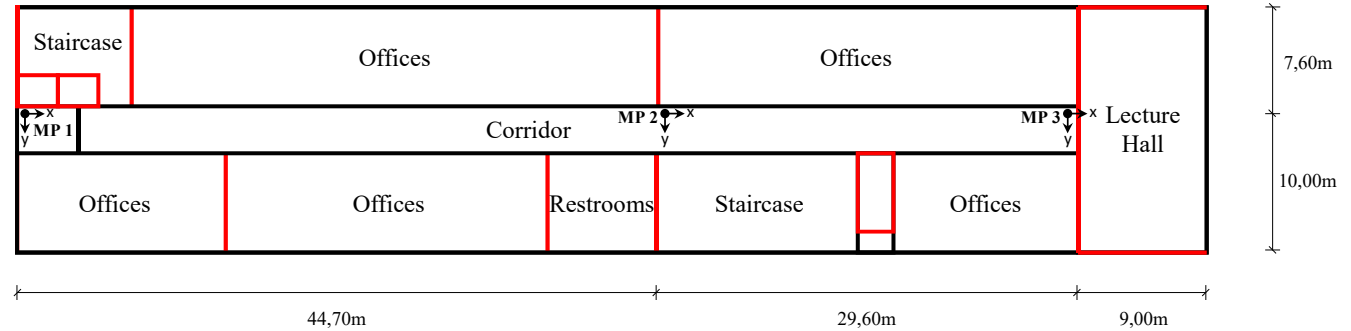

FIgURE 4. Floor plan of the story 6 of the RC-building.

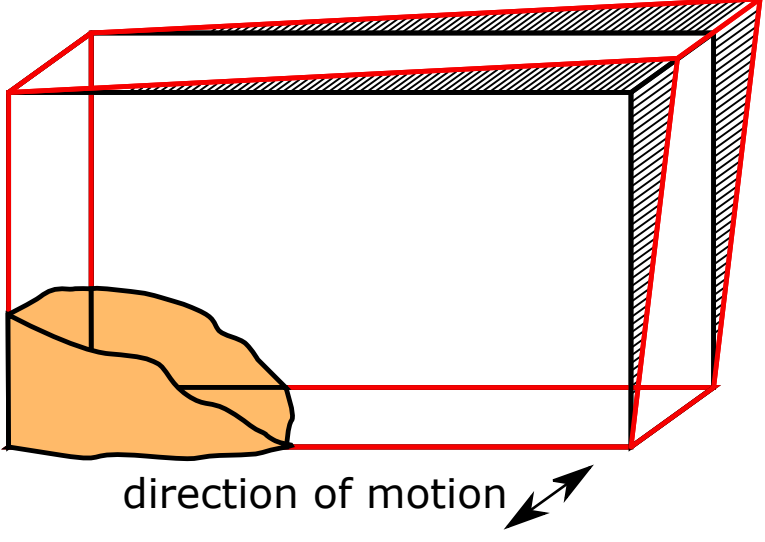

FiguRE $5.1^{\text {st }}$ modal shapes of 7 -story RC-building identified by using FDD.

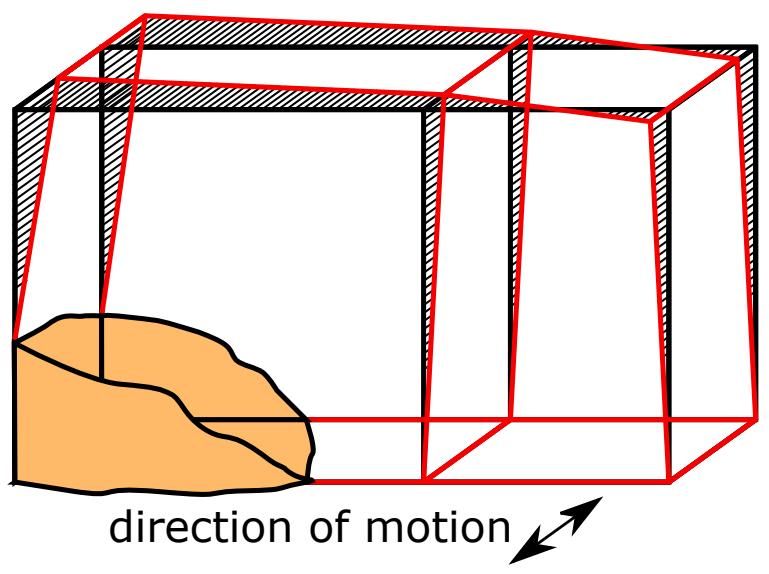

FiguRE $6.2^{\text {nd }}$ modal shapes of 7 -story RC-building identified by using FDD.

\section{Conclusion}

In this paper different system identification methods for civil engineering are introduced and verified both on numerical and experimental examples. The three presented models are the Frequency Domain Decomposition (FDD), the Autoregressive Moving Average Models (ARMA) and the Autoregressive Models with eXogenous input (ARX), which either use the time domain or the frequency domain. Special attention is paid to the type and existence of the excitation signal, which has an influence on the models and the accu-

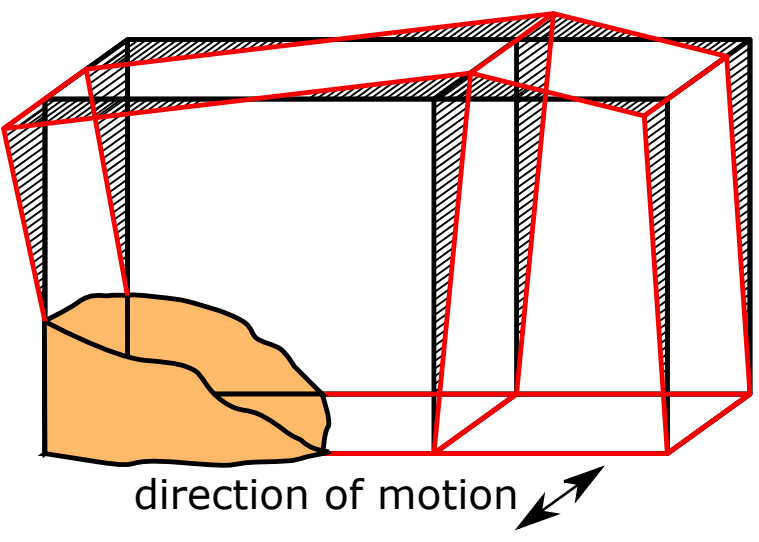

FiguRE $7.3^{\text {rd }}$ modal shapes of 7-story RC-building identified by using FDD.

racy of the results. It is found that the ARMA-model achieves less exact results than other methods, so that they should be preferred, if they are applicable. The first validation example presents the system identification of a numerical 4-dof system model, yielding good agreement with the FE-values. Second example shows the results of an experimental investigation carried out on the 6 th floor of a 7-story RC-building, which allows a preliminary identification of its behavior.

\section{Future Work}

In order to increase the applicability of the system identification methods for civil engineering structures some further research goals need to be accomplished. The algorithms used so far are valid for simple structures without considering the interaction of multiple directions. For real structure applications these methods should be transferred to complex models by modifying the algorithms.

A further innovative approach would be the stepwise investigation of a building, e.g. ambient system responses are measured for several points on separate time windows. The time dependence of the measured data can be corrected by using signal processing methods. Hence compared to other methods the "incremental identification method" (IIM) is a low-cost method, which needs a less extensive measurement preparation by using only one sensor with a numerically affordable data evaluation algorithm. 


\section{ACKNOWLEDGEMENTS}

The authors gratefully acknowledge the financial support of the Daimler and Benz Foundation.

\section{REFERENCES}

[1] R. Brincker, L. Zhang, P. Andersen. Modal identification from ambient responses using frequency domain decomposition. Proc of the Int Modal Analysis Conf IMAC XVIII 2000.

[2] R. Brincker, C. E. Ventura, P. Andersen. Damping estimation by frequency domain decomposition. Proc of the Int Modal Analysis Conf IMAC XVIV 2001.
[3] N.-J. Jacobsen, P. Andersen, R. Brincker. Applications of frequency domain curve-fitting in the efdd technique. Proc of the Int Modal Analysis Conf IMAC XXVI 2008.

[4] J. He, Z.-F. Fu. Modal Analysis. Butterworth Heinemann, Oxford, 2001.

[5] L. Ljung. System Identification. Prentice Hall, New Jersey, 1987.

[6] RWTH Aachen University. 